\title{
REVISIÓN DE LAS EXPERIENCIAS EN EL TRATAMIENTO DE AGUAS RESIDUALES DOMÉSTICAS MEDIANTE REACTORES UASB EN COCHABAMBA-BOLIVIA COMPARADAS CON LAS DE LATINOAMÉRICA, INDIA Y EUROPA
}

\author{
Vanessa Gandarillas R., Oliver Saavedra, Ramiro Escalera y Renato Montoya
}

\section{RESUMEN}

El proceso de tratamiento anaeróbico es cada vez más reconocido como opción viable para la protección del medio ambiente y la conservación de los recursos y representa, combinado con otros métodos adecuados, un sistema de tratamiento de aguas residuales sostenible y apropiado para los países en vías de desarrollo. El tratamiento anaeróbico de aguas residuales se está utilizando con éxito en los países tropicales desde la década de los 80, y con resultados alentadores en las regiones subtropicales y templadas. En este estudio se revisan las principales características del tratamiento de aguas residuales domésticas, con énfasis en el Reactor Anaeróbico de Mantos de Lodos de Flujo Ascendente (RALF) o Upflow Anaerobic Sludge Blanket (UASB de su sigla en inglés). Se revisaron las aplicaciones del proceso UASB en Europa, Asia y las Américas. En Latino América y en particular en Bolivia el uso de esta tecnología ha incrementado considerablemente debido a la pequeña demanda de terreno requerida para su implementación y a sus atractivos costos de inversión y operación. La revisión mostró, en base a la experiencia de operación de distintas plantas de tratamiento ubicadas en los valles de Cochabamba, que los reactores anaerobios de mantos de lodos de flujo ascendente apropiadamente diseñados, son adecuados para el tratamiento de aguas residuales domésticas en las regiones de los valles y llanos de Bolivia debido a que presentan condiciones ambientales que hacen que el uso de esta biotecnología anaerobia sea favorable bajo la perspectiva del desarrollo sostenible.

Palabras clave: Digestión Anaeróbica, Agua Residual Doméstica, UASB, Tecnologías en Plantas de Tratamiento.

DOI: $10.23881 /$ idupbo.017.1-7i 